\title{
Importance of medical data preprocessing in predictive modeling and risk factor discovery for the frailty syndrome
}

\author{
Andreas Philipp Hassler ${ }^{1,3}$ (D), Ernestina Menasalvas ${ }^{3}$, Francisco José García-García ${ }^{4}$, \\ Leocadio Rodríguez-Mañas ${ }^{5}$ and Andreas Holzinger ${ }^{1,2^{*}}$
}

\begin{abstract}
Background: Increasing life expectancy results in more elderly people struggling with age related diseases and functional conditions. This poses huge challenges towards establishing new approaches for maintaining health at a higher age. An important aspect for age related deterioration of the general patient condition is frailty. The frailty syndrome is associated with a high risk for falls, hospitalization, disability, and finally increased mortality. Using predictive data mining enables the discovery of potential risk factors and can be used as clinical decision support system, which provides the medical doctor with information on the probable clinical patient outcome. This enables the professional to react promptly and to avert likely adverse events in advance.

Methods: Medical data of 474 study participants containing 284 health related parameters, including questionnaire answers, blood parameters and vital parameters from the Toledo Study for Healthy Aging (TSHA) was used. Binary classification models were built in order to distinguish between frail and non-frail study subjects.

Results: Using the available TSHA data and the discovered potential predictors, it was possible to design, develop and evaluate a variety of different predictive models for the frailty syndrome. The best performing model was the support vector machine (SVM, 78.31\%). Moreover, a methodology was developed, making it possible to explore and to use incomplete medical data and further identify potential predictors and enable interpretability.

Conclusions: This work demonstrates that it is feasible to use incomplete, imbalanced medical data for the development of a predictive model for the frailty syndrome. Moreover, potential predictive factors have been discovered, which were clinically approved by the clinicians. Future work will improve prediction accuracy, especially with regard to separating the group of frail patients into frail and pre-frail ones and analyze the differences among them.
\end{abstract}

Keywords: Health data analytics, Data mining, Machine learning, Predictive modeling, Risk factor discovery, Data preprocessing, Missing value imputation, Frailty syndrome

\section{Background}

Demographic predictions for the $21^{\text {st }}$ century [1] show a new scenario characterized by a modest increase in life expectancy, but a significantly greater burden of disability, which will increase the demand for health and care costs and challenge the sustainability of the system. Both the

\footnotetext{
*Correspondence: a.holzinger@hci-kdd.org

${ }^{1}$ Holzinger Group, HCI-KDD, Institute for Medical Informatics/Statistics, Medical University Graz, 8036 Graz, Austria

${ }^{2}$ Institute of Interactive Systems and Data Science, Graz University of Technology, 8010 Graz, Austria

Full list of author information is available at the end of the article
}

aging of the population and the growth of the population are driving the increase in Disability Adjusted Life Years (i.e. DALYs) due to the burden of non-communicable diseases in older ages, associated with an increase in years lived with disability. According to the last Global Burden of Disease (2010), disability is the main consequence of the concurrence of the aging process, lifestyles and health conditions [2].

In [1] (and compare also with [3] it is stated that the number of people aged 65+, in Europe, will almost double over the next 50 years, from 85 million in 2008 to 151 million in 2060. This is a great challenge for establishing new 
approaches with more efficient targets for public health and for older people. Hence, the aim is the increase of the life expectancy free of disability and therefore preventing and/or delaying the onset of dependence. This will favor optimization of opportunities for health, participation and security in order to improve quality of life as people age. That is active and healthy aging.

In the field of today's data science there is a wide variety of new and sophisticated computational methods and also tools for building predictive models and performing enhanced data analysis. In clinical medicine these methods are used to offer support in tasks such as decision making based on the patient's data. This covers the spectrum of diagnostic, therapeutic and monitoring tasks. Previous collected patient data can be used to build a predictive model which provides a prediction for the clinical outcome. Clinicians can act on this information and promptly react to possible or likely adverse events [4].

Such an adverse event is for example the onset of frailty, which in [5] is defined as a clinical geriatric syndrome (a more detailed explanation can be found in the subsection Frailty in Related Work). Frailty is characterized by a decreasing capacity to respond to demands of daily life, caused by diminishing functional reserve. The prevalence of frailty in people $65+$ ranges from 7 to $16.3 \%$, increasing with age, and it is the main risk factor for disability [6]. Therefore, frailty assessment is a key tool for the prevention of disability by identification of people at risk.

Data analytics can of course also be applied to analyze retrospective clinical data of the aging population which can be crudely separated into healthy and frail people. This, in order to help to find early predictors for frailty, which in turn would enable the creation of policies for early prevention and adequate early on treatment of the frailty syndrome. Furthermore, this may undoubtedly have a high beneficial impact on society. Sure enough this undertaking, in order to be fruitful, requires extensive medical records of elderly patients.

\section{Objectives}

The main aim of the present work is to demonstrate that data science applied to medical data of elderly, partly frail people can help to find new potential predictors and to obtain a predictive model for the frailty syndrome.

In order to fulfill this goal we will focus on:

1 Building models that are able to discriminate between frail and non-frail people

2 Finding potential predictive factors for frailty

In fact we propose just to focus on frail subjects understood as any people either with the status pre-frail or frail according to the Fried scale [5].

\section{Related work}

The main focus of this paper lies in building predictive models for the frailty syndrome and in discovering potential predictors. Consequently, it will be reviewed in what follows, the existing literature related to data mining in the medical domain and frailty.

\section{Data mining in the medical domain}

Predictive data mining is becoming an important analytical instrument for the scientific community and clinical practitioners in the field of medicine [4]. Secondary use of patient and clinical study data is able to enhance health care experiences for individuals. Further, it enables the expansion of knowledge about diseases and treatments and leads to an increase of efficiency and effectiveness of health care systems [7]. Moreover, molecular data holds the potential to offer insights on single patients, therefore changing decision-making strategies. Thus, it seems predictive data mining will be a strong ally for the transformation of medicine from population-based to personalized practice.

Medical data has already successfully been used for developing various clinical decision support systems' (CDSSs), which significantly impact practitioner's performance and the health care process in a positive way and will do so in the future $[8,9]$. Nevertheless, there still is a lot of room for improvement and the remaining issues have to be tackled.

Regarding building predictive models, the currently widely used neural networks (NN) [10] and also the deep learning approaches [11] are a very robust group of techniques with a good performance and they do deliver very promising results, but they are very hard to interpret because of their complex inner working. Simpler techniques like the naive Bayes classifier (NB) [12], linear discriminant analysis (LDA) [13], support vector machines (SVM) [14] and tree-based approaches [15] produce results that are much easier to interpret. Consequently, we propose in this paper to use the latter kind of techniques.

An important feature of the medical data due to its nature, is that in order to understand it, the involvement of the medical professional is paramount. Interactive machine learning (iML) [16] approaches allow to insert the physician in the "loop" of learning and that is what we have attempted to realize in this research.

\section{Frailty}

The frailty syndrome was defined by Fried et al. [5] as a syndrome where three or more of the following criteria are present: unintentional weight loss $(10 \mathrm{lbs} / 4.54 \mathrm{~kg}$ in the past year), self-reported exhaustion, weakness (measured via grip strength), slow walking speed, and low physical activity. Subjects with no deficits in all criteria score 0 , 
which means they are not frail. Those who have deficits in 1 criterion or 2 criteria are called intermediate frail or pre-frail. All higher scores lead to the classification frail.

Frailty is considered highly prevalent in old age and associated with an elevated risk for falls, disability, institutionalization, hospitalization, and mortality [5]. However, it should not be considered synonymous with disability or comorbidity. Fried et al. state that comorbidity should rather be treated as an etiologic risk factor for frailty and disability as an outcome. Disability cannot be reversed, but it is preceded, sometimes by several years, by the frailty syndrome, which can be reversed, and thus prevented from worsening and its progression monitored.

Even that we use this work [5] as reference, in this research also other literature regarding frailty is presented. Apart from the index Fried et al. proposed, also others have emerged $[17,18]$. Moreover, frailty is entangled with other concepts like disability and comorbidity and some effort has already been made to separate those [19]. Frailty has been also successfully used as a predictor itself, for example for predicting postoperative outcomes [20], where one study [21] found that it is more useful than conventional methods. These findings affirm the potential of the syndrome definitions and available indexes as being a stable concept.

Frailty seems to be strongly connected to physical activity and exercise, which have been proven to be protective factors [22, 23]. Further, it seems that the syndrome is closely related to mental impairment and mental health, especially depression [24]. Increased age and not having a daily consumption of vegetables and fruits were each associated with frailty or pre-frailty [25]. There is also a considerable gender aspect to this syndrome. Women are more likely to become frail in higher age and also frail women have a higher risk of developing disability, being hospitalized and death [26]. Moreover, some physiological blood parameters seem to be related to frailty and hold the potential to serve as markers and/or predictors. Studies found that this geriatric syndrome is also related to increased inflammation and elevated markers of blood clotting [27]. In a study done by Baylis et al. (2013) [28] the relationship between immune-endocrine parameters and frailty and also mortality after 10 years in females and males with an age between 65 and 70 years was investigated. Their findings were that higher baseline levels of white blood cell counts, lower levels of dehydroepiandrosterone sulfate (DHEAS) and higher cortisol to DHEAS ratio could be related to a higher probability of frailty in the future. Additionally, it was found that the presence of diabetes also is a risk factor for the onset of the frailty syndrome [25]. Concluding, a lot of suitable predictors (preventive and risk factors) have already been found and are used for frailty screening and also prediction.
From the previous review of literature related to the frailty syndrome the main conclusions are:

1 Fried's frailty score [5] seems to be the one widely-used by physicians

2 In the research of Fried et al. the following factors are used to establish the frailty level (non-frail, pre-frail and frail):
(a) unintentional weight loss (10 lbs in past year)
(b) self-reported exhaustion
(c) weakness (grip strength)
(d) slow walking speed
(e) low physical activity.

These variables are highly correlated with the variable presenting the frailty status. Thus, we propose in our research to use any other factors (variables) to predict frailty.

\section{Methods \\ Data}

We used the data of the Toledo Study for Healthy Aging (TSHA). In [29] the TSHA is described as follows. The Toledo study is a population-based study conducted on 2488 individuals aged 65 years and older. The study subjects were selected by a two-stage random sampling from the Toledo region. Institutionalized as well as community dwelling persons were selected. Data was gathered in 3 waves: first (2006 to 2009) information on social support, activities of daily living, comorbidity, physical activity, quality of life, depression symptoms, and cognitive function was collected. Furthermore, anthropometric data (mass and length of body segments) and results of physical performance tests (walking speed, upper extremity and lower extremity strength, and the stand-and-sit from a chair test) were collected and a blood sample was obtained. Many of the used variables are also recommended by the American Geriatrics Society (AGS) for screening older patients for risk of falling and preventing falls. The diagnosis of the frailty syndrome was based on the Fried criteria (weakness, low speed, low physical activity, exhaustion, and weight loss)[5]. In the second wave (2011-2013) and in the third wave (2015-2017), which is ongoing, additional parameters were added (urine parameters). In the first wave the data of 474 patients was available, of which remained 354 in the second wave.

From the aforementioned Toledo study a subset of data has been made available for this work. In particular, anonymized data of 474 patients has been provided. Thereby, for each patient medical data consisting of 284 parameters was available. The majority of attributes belong to the first wave of the TSHA (2006-2009) and only 21 come from the second study wave conducted in 2011-2013. 


\section{Definition of the variables}

The provided data set contains 284 variables from which only one is considered the variable to be predicted, the FRAILTY variable. Even that in the TSHA study this variable takes the values: non-frail, pre-frail and frail, for the present study the variable is a binary one in which the classes pre-frail and frail have been fused together. Therefore, the in this work used target variable FRAILTY consists of the classes non-frail (value: 0 ) and frail (value: 1 ).

Hence, in total 180 observations are non-frail and 294 observations are frail. The remaining 283 variables will be used to build models for the FRAILTY variable. They were grouped according to their semantics into: i) demographic, ii) phenotype, iii) medication and iv) code features. The phenotype features then were further split into physique, blood, cardiac, disease, self reported disease, consumption and medical test attributes.The medical test attributes were further divided into features corresponding to the Geriatric Depression Scale (GDS), Activities of Daily Living (ADL), Instrumental Activities of Daily Living (IADL), Mini-Mental-State-Examination (MMSE) and Mobility Scale (MS) attributes.

Below you can find a short explanation for each medical test, which was carried out in the TSHA [29]:

Geriatric depression scale (GDS) This scale was created with the objective to obtain a reliable rating for depression in elderly. The applicant himself answers in the so called short form 15 different questions. Of those, 10 questions indicate the presence of depression when positively answered and the remaining 5 questions indicate the presence of depression when negatively answered. The test yields a score between 0 and 15, where scores between 0 and 5 mean no depression is present and values above 5 indicate the presence of a depression [30, 31].

Activities of daily living (ADL) In this assessment also a questionnaire is used, which is answered by the patient. Here the goal is to estimate the patients' satisfaction in his daily activities, which contain hygiene, alimentation and independent access to necessities. There exist different variations of the ADL test, which differ regarding their contained number of questions. In this work the ADL according to Katz [32] was used. The answers to 6 different questions provides a score between 0 and 6 , where a score of 0 signifies no ability of self-care and a score of 6 complete ability of self-care.

Instrumental activities of daily living (IADL) Like the ADL-test but mainly focused on instrumental activities. These include following daily tasks and responsibilities: food preparation, shopping, using the telephone, housekeeping, transportation, responsibility for own medications and the ability to handle finances. For each activity exist 3 to 5 questions, each yielding 0 or 1 point. The maximum for each category is 1 point and signifies that the ability to perform that certain task is given. At the end these points are summed up. This sum represents the IADL-Score with a range between 0 and 8. [33]

Mini-mental-state-examination (MMSE) The MiniMental-State-Examination represents standardized test for cognitive function or measure of impaired thinking. The tested areas of cognitive function consist of orientation, registration, naming recall, calculation, writing, attention, repetition, comprehension, reading and drawing. The range of the result lies between total cognitive absence ( 0 points) and full cognitive function (30 points) [34, 35].

Mobility score (MS) The MS questions belong to the Physical Activity Scale for the Elderly (PASE) questionnaire [36]. They provide validated knowledge about the physical activity of the patients. Here, 5 principal questions and follow-up questions were asked, yielding to a in this work derived score between 0 and 5 . The maximum score indicates full mobility and 0 signifies extremely limited mobility.

\section{Data exploration and quality assessment}

The retrieved data set was analyzed using different statistical visualization techniques like plotting the histogram, the kernel density function estimate and box-plots. Further, the values of each feature were inspected and compared to the values they should have according to the provided data dictionary (Additional file 1). Moreover, statistical measures were calculated and analyzed. The provided variables were divided according to their corresponding data type into continuous, categorical and binary variables. Depending on this data type, different visualizations were realized and statistical measures calculated. Features representing codes and IDs of the TSHA data do not contain relevant information with regard to frailty prediction, as they were created for organizational reasons and do not contain information regarding medical/phenotypic/demographic aspects.

In total the data set contains 474 observations and 284 features including the target variable representing the frailty status. In total 176 features are more than $90 \%$ complete and in 41 features more than $50 \%$ of the values are missing, of which 12 are follow up questions to a previous asked principal question. For example the feature $t a b 1$ contains the answers to the question "Have you smoked at least 100 cigarettes in your entire life?", when answered with 2 (which stands for no) the follow up question, represented by tab1a ("If yes, Did you smoke cigarettes daily, occasionally, or not at all?"), has not been asked. So as a matter of fact, these values are not missing at random, but 
rather the question was not applicable for these observations. One can see that in order to make use of all the observations and therefore of the contained information, a special strategy for dealing with missing data is clearly necessary. For many features a special treatment is necessary in order to better capture their actual meaning as the current values do not sufficiently reflect it.

Through analysis of known frailty related factors via ontology-guided PCA using the approach described by Wartner et al. (2016) [37], mining association rules using the apriori algorithm [38] and general correlation statistics, it can be assumed that the from the doctors described relationships are also present in the data.

\section{Data preparation}

In this phase the data is cleaned, prepared and when necessary transformed. Further, new features are derived and the quality of the features in terms of predictiveness is assessed.

First the features are analyzed regarding their contained information in a statistical view and in a semantic view. In the scope of this work it was decided to exclude information regarding drugs. On the one hand because the information presented is not sufficiently structured and the pre-processing required exceeds the time available for this work and on the other hand, because doctors preferred to have the first predictive model only with phenotypical parameters and results of the different tests.

Features which belong to the follow-up study conducted in the years 2011-2013, were discarded, as there were only 21 of them (and the remaining 264 are from the earlier study wave) and therefore a temporal analysis was not possible. Also features, which in a statistical sense contain no information, were excluded. An example therefore is the feature that describes binarily the presence of leukemia or polycythemia. As all the observations have the same value "2" (meaning "not present"), this feature was excluded. Summing up, a total of 196 variables were left for further analysis.

The data set was inspected regarding potential outliers, using the reference variable ranges according to the data dictionary (Additional file 1). Not described appearing values were examined from a statistical point of view using the informal box plot method. Additionally, the kernel density estimate was analyzed. After that exploration, domain-knowledge was used to analyze the significance of appearing extreme values. Further, the doctors of the hospital were involved in the decision if the values are plausible and should be kept, or if they should be discarded. Moreover, possible/plausible values were discussed with them and thresholds were established, exceeding values then simply were set to not available (NA).

After the data had been cleaned, the following step was to extend the available data set by creating new features, using the available ones. Sometimes the doctors can provide some scores or ideas for building features. A simple example for this purpose is the Body Mass Index (BMI) [39], which can be easily calculated using the patients weight in $\mathrm{kg}$ and his height in $\mathrm{cm}$ (see Eq. 1).

$$
B M I=\frac{\text { weight }}{\left(\frac{\text { height }}{100}\right)^{2}}
$$

Other than the BMI also features representing the mobility score (sum of the answers to the principal questions of the mobility score, see subsection Definition of the Variables) and the total income (sum of household income and individual income) were created.

\section{Imputation of missing data}

Once data is prepared and new features have been derived, the following step is to make sure all the observations can be used in the modeling phase. Therefore, it was decided to calculate different estimates for each missing value. Thus, missing values are imputed (filled) with estimates.

In Table 1 the features where more than $5 \%$ of the values are missing can be seen. These measures are referring to the already in the previous steps pre-processed data set. An important step before applying imputation techniques, is to assess the reason for missingness. Three types of missing data exist and they are called Missing Completely At Random (MCAR), Missing At Random (MAR) and Missing Not At Random (MNAR). The assumed reason for the missingness and the according applicability of imputation techniques is also presented in Table 1. Features where more than one third of the values are missing were excluded from further investigations. They are marked in bold. Overall, all MNAR cases can be found in features which represent follow-up questions, they therefore were only be answered if the underlying basis question was answered positively. For them no imputation is possible because they can't be derived from other features.

In order to use all the available information contained in the data set, different imputation settings using the MICE implementation, more specifically the CALIBERrfimpute [40] expansion of it, were considered. Following configuration, regarding the imputation method, was chosen:

- For continuous features: rfcont for numeric random forest (RF) imputations

- For binary, ordered and unordered categorical features: rfcat for categorical RF imputations (factor, $\geq 2$ levels)

Due to the size of the data set and the high number of features it was decided to use a selection of suitable features for the imputation models. One way is selecting manually every predictor for every imputation model and another 
Table 1 Overview of features with more than $5 \%$ missing values

\begin{tabular}{|c|c|c|c|}
\hline Feature & Percentage of missing data & Reason for missingness & Imputation possible \\
\hline Times stopped smoking & 75.11 & MNAR (follow-up question) & No \\
\hline Daily wine consumption & 91.14 & MNAR (follow-up question) & No \\
\hline Daily beer consumption & 98.73 & MNAR (follow-up question) & No \\
\hline Daily spirits consumption & 98.95 & MNAR (follow-up question) & No \\
\hline Duration of alcohol consumption & 82.91 & MNAR (follow-up question) & No \\
\hline Earlier alcohol consumption & 19.20 & MAR & Yes \\
\hline Kind of drinker (earlier) & 86.29 & MNAR (follow-up question) & No \\
\hline Starting age alcohol consumption & 86.50 & MNAR (follow-up question) & No \\
\hline Ending age alcohol consumption & 86.92 & MNAR (follow-up question) & No \\
\hline $\mathrm{D}$ Dimer $[\mu \mathrm{g} / \mathrm{L}]$ & 17.72 & MAR & Yes \\
\hline High-sensitivity C-reactive protein (hs-CRP) [mg/L] & 14.98 & MAR & Yes \\
\hline Number of IADL abilities & 6.33 & MAR & Yes \\
\hline Total MMSE score & 15.82 & MAR & Yes \\
\hline Total GDS & 9.49 & MAR & Yes \\
\hline Depression & 9.49 & related to $\{$ gdstotal\} & No \\
\hline Insulin [U/mL] & 11.60 & MAR & Yes \\
\hline HDL & 9.07 & MAR & Yes \\
\hline LDL & 9.07 & MAR & Yes \\
\hline Total testosterone [ng/dL] & 37.97 & MAR & Yes \\
\hline Free testosterone $[\mathrm{ng} / \mathrm{dL}]$ & 37.97 & MAR & Yes \\
\hline Mobility scale question 5 & 8.44 & MNAR (follow-up question) & No \\
\hline Mobility scale question 6 & 8.44 & MNAR (follow-up question) & No \\
\hline Mobility scale question 8 & 14.35 & MNAR (follow-up question) & No \\
\hline Mobility scale question 9 & 13.92 & MNAR (follow-up question) & No \\
\hline Mobility scale question 11 & 7.81 & MNAR (follow-up question) & No \\
\hline Mobility scale question 12 & 7.59 & MNAR (follow-up question) & No \\
\hline Mobility scale question 14 & 25.95 & MNAR (follow-up question) & No \\
\hline Mobility scale question 15 & 26.16 & MNAR (follow-up question) & No \\
\hline MMSE temporal domain 1 & 17.93 & MAR & Yes \\
\hline MMSE temporal domain 2 & 18.78 & MAR & Yes \\
\hline MMSE temporal domain 3 & 18.14 & MAR & Yes \\
\hline MMSE temporal domain 4 & 22.57 & MAR & Yes \\
\hline MMSE temporal domain 5 & 12.87 & MAR & Yes \\
\hline MMSE spatial domain 1 & 13.08 & MAR & Yes \\
\hline MMSE spatial domain 2 & 13.29 & MAR & Yes \\
\hline MMSE spatial domain 3 & 13.29 & MAR & Yes \\
\hline MMSE spatial domain 4 & 13.29 & MAR & Yes \\
\hline MMSE spatial domain 5 & 13.29 & MAR & Yes \\
\hline MMSE remembering 1 & 18.99 & MAR & Yes \\
\hline MMSE remembering 2 & 19.41 & MAR & Yes \\
\hline MMSE backward counting & 51.05 & MAR & Yes \\
\hline MMSE spell the word & 61.60 & MAR & Yes \\
\hline MMSE object naming & 13.92 & MAR & Yes \\
\hline MMSE repeat phrase & 13.08 & MAR & Yes \\
\hline MMSE left right & 13.50 & MAR & Yes \\
\hline
\end{tabular}


Table 1 Overview of features with more than 5\% missing values (Continued)

\begin{tabular}{llll}
\hline Feature & Percentage of missing data & Reason for missingness & Imputation possible \\
\hline MMSE following written order & 13.29 & MAR & Yes \\
MMSE write sentence & 13.92 & MAR & Yes \\
MMSE copying design & 13.50 & MAR & Yes \\
Cognitive impairment & 17.09 & MAR & Yes \\
Individual income & 8.44 & MAR & Yes \\
Household income & 13.29 & MAR & Yes \\
Number of persons in the family & 18.78 & MAR & Yes \\
Insulin like growth factor 1 (IGF1) [ng/mL] & 27.00 & MAR & Yes \\
Dementia type & $\mathbf{9 8 . 7 3}$ & MNAR (follow-up question) & No \\
Overall income & 13.71 & MAR & Yes
\end{tabular}

Features where more than one third of the values are missing are presented in bold

way is to use statistical measures for the selection. Consequently, is it for example possible to just consider variables which show a correlation higher than a certain specified percentage. As it is a rule to use as much information as possible as this leads to multiple imputations which have a minimal bias and a maximal certainty [41], a minimal correlation-threshold of $7 \%$ was used.

Additionally, only such variables which are more than a certain desired percentage complete will be used. For the first imputation only predictors, which correlate more than $7 \%$ and are more than $80 \%$ complete were selected by configuring the parameter pred. The overall configuration of the mice() function can be seen in following code-fragment.

mice (data, seed $=219$, pred $=$ quickpred $(\mathrm{imp}, \operatorname{mincor}=0.07$, minpuc $=0.8)$,

defaultMethod = c("rfcont ", "rfcat ", "

rfcat ", "rfcat "),

$\mathrm{m}=5$, maxit $=70$, MaxNWts $=9000)$

Here, MaxNWts depicts the maximal number of weights used by the inner neural network. The argument maxit was used to set the maximal numbers of iterations to 70 . As creating 5 different imputations was desired, the parameter $m$ was set to 5 . The argument defaultMethod contains the different per default used methods for the different data types, which were already mentioned earlier. Using pred, different restrictions regarding minimum correlation and completeness of the predictors were added. The first argument represents the data set in matrix form for which the imputations should be computed. The parameter seed can be used to set the number for initializing the pseudo-random generator.

The mean and the standard deviation for each variable at each iteration can be observed in the received imputation object. These values were plotted for the features with the highest amount of missing values in order to see if median and variance of the different imputations do converge. It seemed that 70 iterations are quite sufficient in this regard.

The obtained imputations are then examined using visualization tools. One possibility to check if the obtained imputations are reasonable, is to compare the kernel density estimates of the observed and the imputed values for ideally all variables. As this would not have been feasible within the scope of this work, only features with more than $5 \%$ missing values were examined. Further, the kernel-density function was plotted and analyzed for each feature and each imputation in order to evaluate the quality.

The second imputation was done the same way, but this time also the selected features were included for every imputation model. This is recommended by Buuren and Groothuis-Oudshoorn (2011) [41]. The connection between the imputation and the feature selection process is demonstrated in Fig. 1.

The overall configuration of the mice() function for the second imputation can be seen in following codefragment.

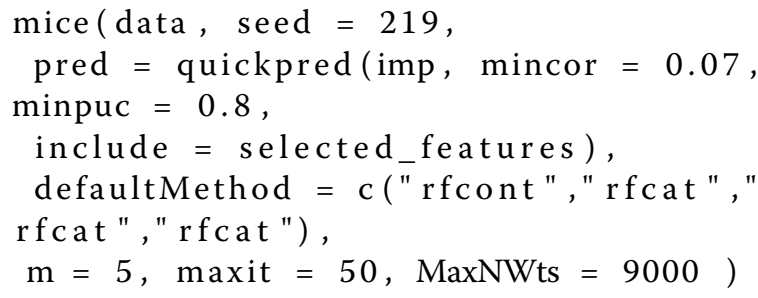

The only difference is that by adding the parameter include = selected_features to the attribute pred, the selected features are used additionally for every imputation model.

Here, the obtained imputations were also analyzed as it has been done before. With the help of density plots of the imputed and the original values, once again the quality 


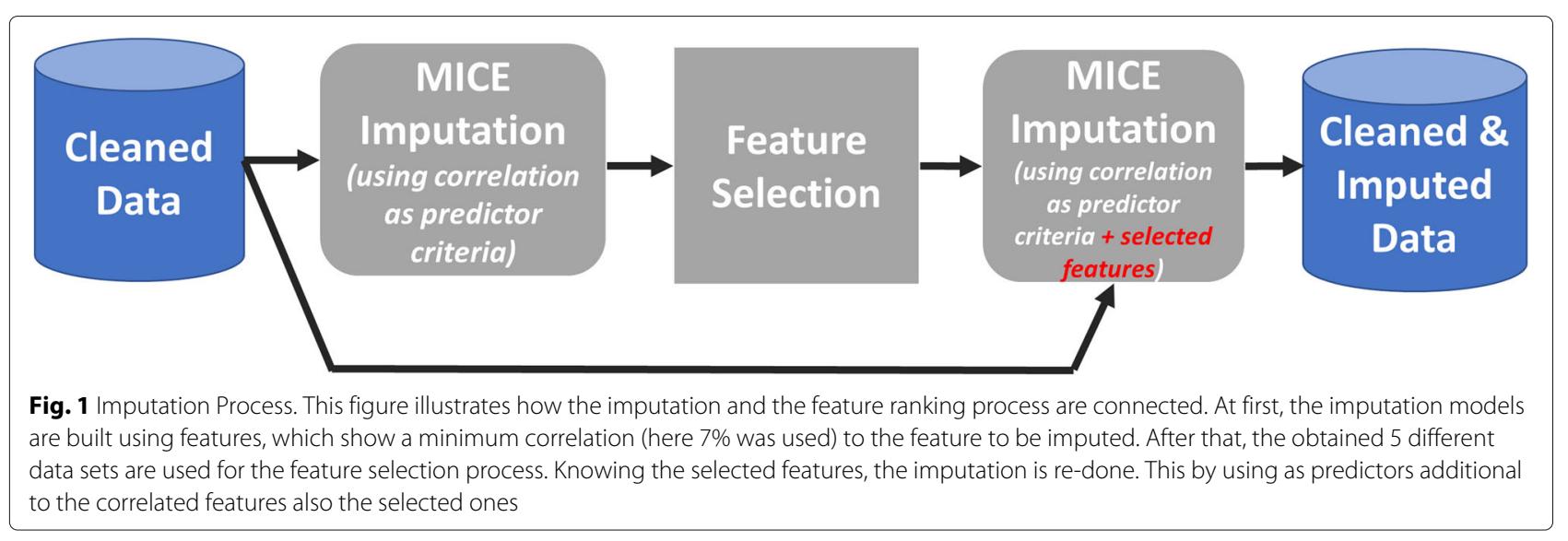

of the imputations was assessed. The obtained 5 different imputed data sets then were used for the modeling process.

\section{Feature selection}

As the objective is to predict the FRAILTY variable with a subset of features, which are highly predictive, the most predictive features were determined using feature ranking methods. Further, the obtained results were compared with the suggested factors from the doctors of the Toledo study.

In order to make just use of the features which are indeed predictive and therefore beneficial for the final predictive model in terms of performance, different feature selection methods were considered. Finally, it was decided to use the Boruta [42] algorithm, which uses a RF wrapper method. The implementation of the R package Boruta [42] was used. The selection was performed with regard to the binary target variable FRAILTY.

For each imputed data set the feature selection process using the Boruta algorithm was executed. For the sake of obtaining reliable and stable results, the method was configured to use 1000 trees for the RF algorithm and to perform 1000 runs in order to avoid so called tentative results. This means there are still features, which could not be rejected nor accepted for the final set. At the end, 5 different sets of selected features were present. The finally chosen selected features were those, which appeared at least 3 times in the 5 different Boruta sets. The complete feature selection process, which begins after the first executed imputation procedure and provides the selected features for the second imputation, is shown in Fig. 2.

Findings In Fig. 3 the result of the feature selection is presented. The variables are ordered by importance, the rejected ones are colored red, the selected ones green and those, for which no decision could be made, are yellow. All the importance measures of the features were compared to randomly permuted copies of themselves, so called shadow attributes. The Z-Score of the most important shadow attribute was used as separator between selected and rejected features. Features where no decision could be made were marked tentative and colored yellow.

By using the function TentativeRoughFix those features, with a median importance higher than the maximal one of the shadow attributes, were selected and the others rejected. This is a simple test for judging these tentative attributes. Tentative attributes could also be resolved by increasing the number of importance runs of the Boruta algorithm. That is why instead of the default 100 runs, 1000 runs were used.

After the feature selection, the obtained final variables were used for another imputation round. As suggested by Buuren and Groothuis-Oudshoorn (2011) [41], the features which are powerful in terms of predictiveness, with regard to the target variable, should always be used in the imputation for each feature. That is why they all were included in each imputation model.

\section{Modeling and evaluation}

Once data had been prepared, the following step was to build predictive models. As can be seen in the sections to come, different techniques have been applied. Later the received results have been compared and validated. In what follows, one can find the model settings, the modeling and validation schema, the model performance and lastly the evaluation of the models.

\section{Classification model settings}

Following learning algorithms for the predictive models have been chosen: the NB algorithm, Classification And Regression Trees (CART), bagging CART, C5.0, RF, SVM and LDA. They have been selected as representative of the most widely used in the literature [43]. This variety of algorithms allows to analyze the robustness of the solution and how different methods influence the performance. The different algorithms were implemented in the R environment using different third party packages, which are 


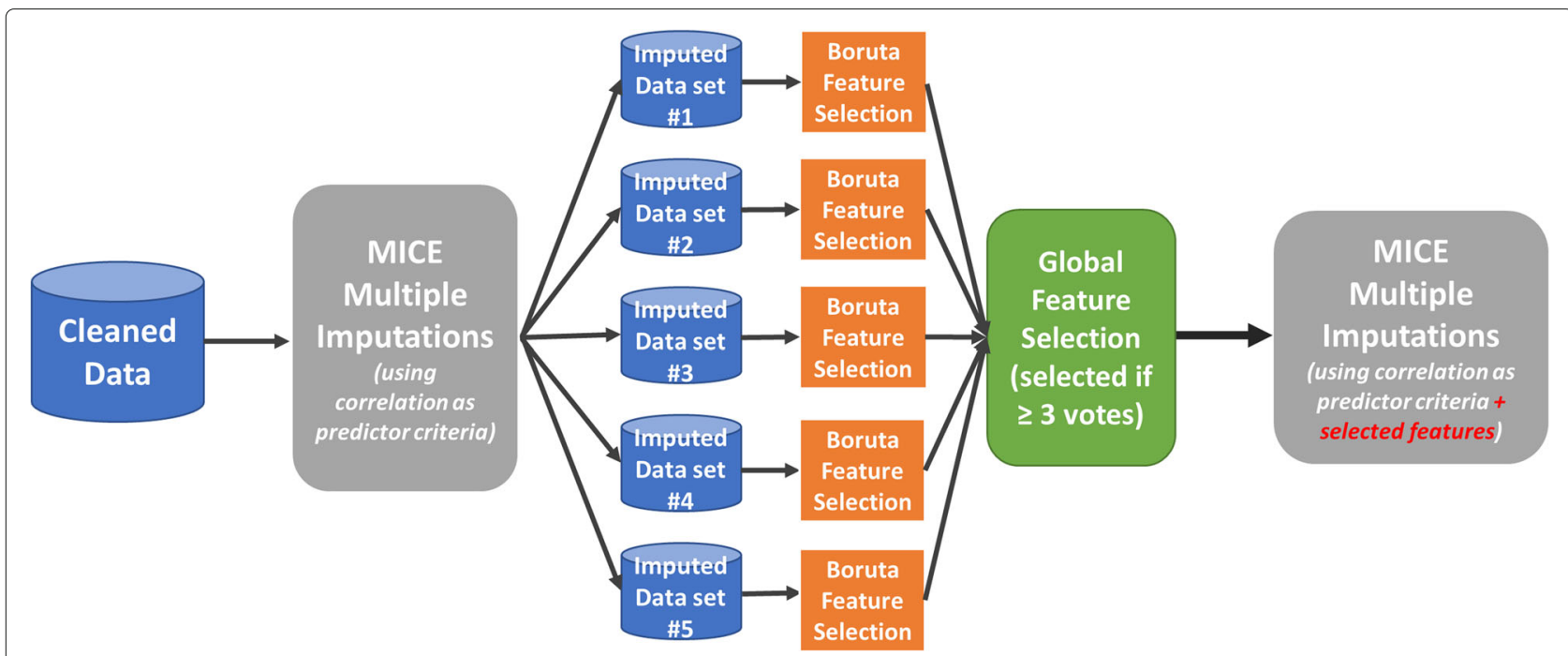

Fig. 2 Feature Selection Process. This figure shows the overall feature selection process. At first, the Boruta algorithm is applied on each imputed data set. Then, the 5 different selected feature sets are compared and features which appear in 3 or more selected sets are chosen for the final feature set

listed in what follows. Further, changed configurations, which differ from the default settings are described in this listing.

NB The NB classifier naiveBayes of the R package e1071 was used in its standard configuration.

CART The CART algorithm tree of the same titled $R$ package was used in it's standard configuration.

Bagging CART The bagging CART implementation bagging from the $\mathrm{R}$ package ipred lead to the best results, when using 55 bootstrap replications.
C5.0 The best accuracy for the C5.0 algorithm (from the $\mathrm{R}$ package $\mathrm{C50}$ ) could be achieved using 50 iterations for the multiclass classification and 55 iterations for the binary classification.

RF The best accuracy in the RF implementation "randomForest" from the R package with the same name was achieved, using 1000 trees, no replacements in the inner sampling of cases and 5 as number of variables randomly sampled as candidates at each split.

SVM The best setting for this algorithm was using as type the C-classification, as kernel the radial basis function (RBF) and as tolerance of termination criterion the value $10^{-3}$. The degree was set to 3 , the ' $\mathrm{C}^{\prime}$-constant of the

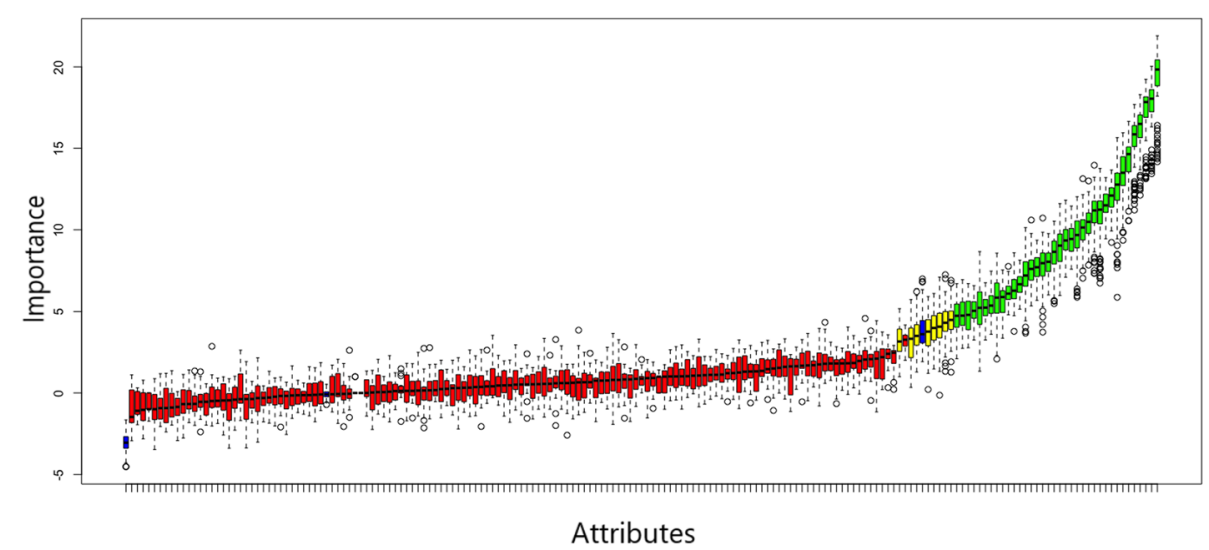

Fig. 3 Feature Selection Results (Boruta). This image shows the attributes and their importance measure, by which they were selected (green) or rejected (red). This decision was made by comparing their importance measure to randomly permuted copies of themselves, the so called shadow attributes [54]. Features which could neither be selected nor rejected were marked tentative (yellow) 
regularization term in the Lagrange formulation was set to 10 and the gamma of the RBF was set to 0.07 .

LDA This method from the R package MASS was used in its standard configuration.

\section{Optimization of algorithm input}

In order to utilize the data in the best way, it has been shown that sometimes it is beneficial for the performance of the learning algorithms to transform the data to different ranges and also to change the distribution. This was also considered in this work and therefore, every algorithm was used on the z-score standardized, the Min-Max normalized and the raw data set. Where the raw form represents the data after completion of the preprocessing phases.

Min-Max Normalization Min-Max normalization is a method where the values of the data are transferred into a range of $[0,1]$. Where the lowest appearing value $x_{\min }$ is set to zero and the maximal value $x_{\max }$ is set to 1 . The used formula is shown in Eq. 2. Here each value $x_{i}$ is Min-Max normalized using its current value, $x_{\min }$ and $x_{\max }$.

$$
m m\left(x_{i}\right)=\frac{x_{i}-x_{\min }}{x_{\max }-x_{\min }}
$$

For each learning algorithm the 3 aforementioned input data set variants were used and the resulting performances were compared. Then for each algorithm the variant which leads to the best performance was chosen.

\section{Modeling and validation schema}

After preparing the data for the modeling phase, the next step was building the models and validating them. In Fig. 4 the procedure for modeling and evaluating is presented. At the beginning each obtained imputed data set is used to build the different models (e.g. RF, DT, SVM), which are tested in a cross-fold validation setup. The resulting performance measure values of each model for each imputation are then compared and the one with the overall best performance is chosen as final model. Therefore, 5 different final models are obtained at the end. Afterwards they can be used as an ensemble classifier, which provides one result for new unseen instances.

In order to evaluate the out of sample error of the built models, as mentioned before, the very commonly used 10-fold cross-validation was performed. Here, the training data is split into 10 different, generally equal-sized folds. Then, for each fold $k$ the model is trained on all the folds but the $k^{\prime}$ th. After that the obtained model is tested on the $k^{\prime}$ th fold. This is repeated for all 10 folds, where $k=1 \ldots 10$. The error averaged over all the folds is then computed.

Due to the fact that the classes are imbalanced (180 observations are non-frail and 294 observations are frail), a stratification technique was implemented. This in order to have the same number of frail and non-frail observations in each created fold and thereby maintaining the initial class balance. At first, the observations were split according to their frailty status (2 classes). Afterwards, the 10 folds were created separately for each class and then fused according to the fold-number. The observations were chosen randomly.

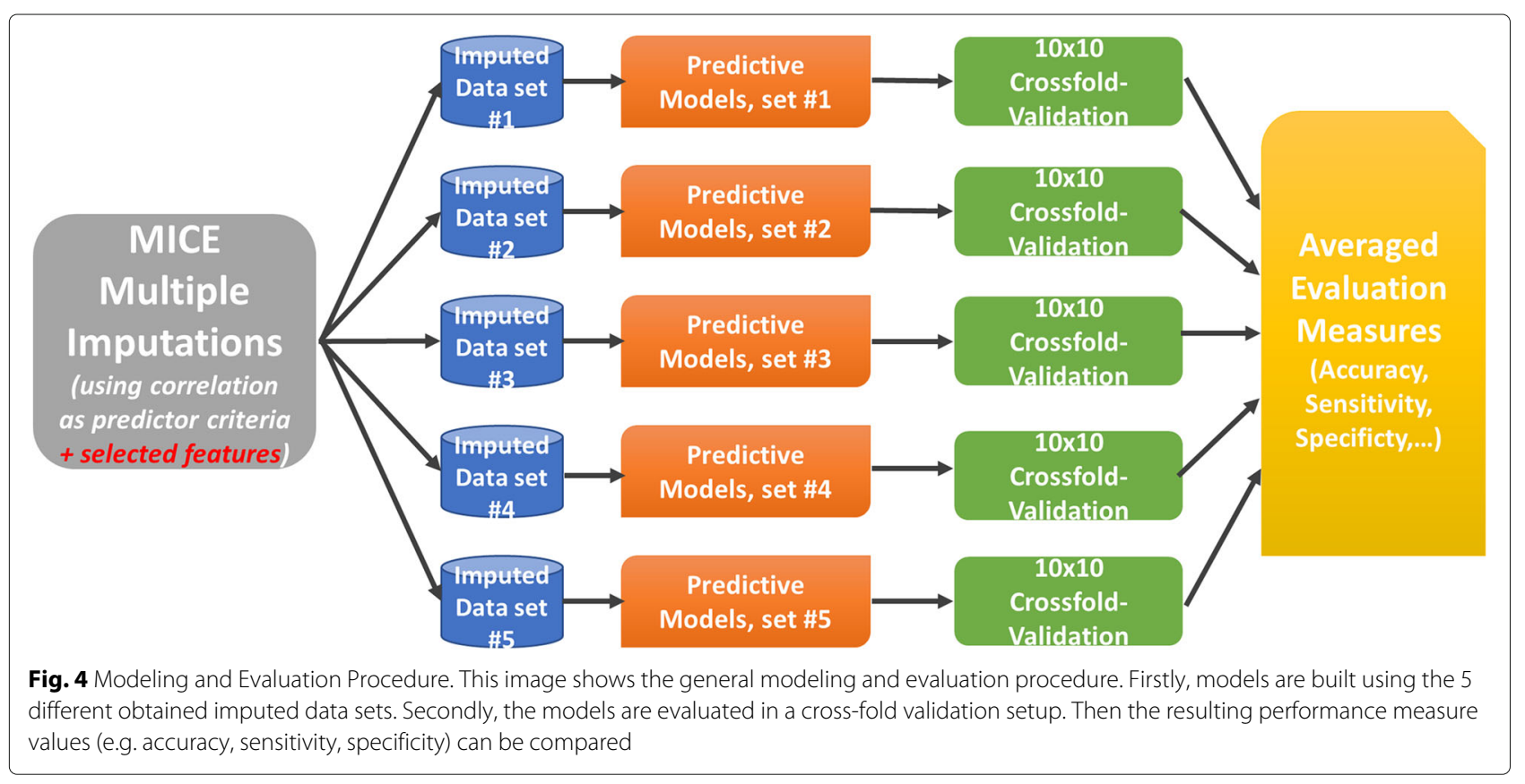


By using multiple 10-fold cross-validations, a first estimate of the generalization error is obtained. For the modeling phase five different imputed data sets were considered, thereby five different best performing classifiers were obtained. The final predictive model represents an ensemble classifier, which can be used on new unseen instances. The final predicted class is the result of five different votes, where each vote is the corresponding classification result of each model.

\section{Performance measures}

The confusion matrix of the prediction results is the basis for the following used measures: accuracy, sensitivity (also called true positive rate), specificity (also called true negative rate), precision, $F_{1}$-Score and the area under the receiver operating characteristic curve (AUC) [44]. A more detailed description of the used performance measures can be found in [45].

\section{Results}

\section{Selected features}

The finally selected features, which appeared at least 3 times in the 5 different feature selection sets, using the Boruta algorithm, can be seen in Table 2 .

\section{Model performance}

The model performances were obtained by averaging each performance measure for the 10 different 10 -fold crossvalidation setups. The obtained results can be seen in Table 3. For each performance measure, the over the folds averaged value including the standard deviation is shown. The highest obtained value for each performance category is marked in bold.

\section{Discussion}

The goal of this work was to build models that are able to discriminate between frail and non-frail people and to find potential predictive factors for frailty using data mining. For this purpose the medical data provided from the TSHA was used.

\section{Data understanding}

The data understanding phase has shown to be useful to understand the relationship between variables and to find outliers, correlations and obtain general insights that have guided later the predictive modeling process. In fact the analysis of all the features helped to determine their particular importance in the frailty prediction. Further, the application of the ontology-based PCA approach described by Wartner et al. (2016) [37] was able to deliver some insights, which were further investigated.

\section{Selected features}

A step of the process that resulted to be especially important was feature selection, given the high number of
Table 2 Obtained final selection of features using the Boruta algorithm and a voting system (presence of the feature in at least 3 out of the 5 sets)

\begin{tabular}{|c|c|}
\hline Description & Type \\
\hline Height (cm) & Numeric \\
\hline Presence of cognitive impairment & Binary \\
\hline Presence of depression & Binary \\
\hline $\begin{array}{l}\text { Mobility Scale follow-up question (tiredness when } \\
\text { going out) }\end{array}$ & Binary \\
\hline Mobility Scale question (stair-climbing ability) & Binary \\
\hline $\begin{array}{l}\text { Mobility Scale follow-up question (tiredness when } \\
\text { walking outside) }\end{array}$ & Binary \\
\hline Mobility Scale question (walking outside ability) & Binary \\
\hline $\begin{array}{l}\text { MMSE follow-up question (remembering objects } \\
\text { ability) }\end{array}$ & Categorical \\
\hline Total GDS & Binary \\
\hline Age in years & Numeric \\
\hline ADL question (difficulty washing) & Categorical \\
\hline Number of ADL abilities & Numeric \\
\hline Number of IADL abilities & Numeric \\
\hline IADL question (difficulty using telephone) & Categorical \\
\hline IADL question (difficulty shopping) & Categorical \\
\hline IADL question (difficulty cooking) & Categorical \\
\hline IADL question (difficulty doing light housework) & Categorical \\
\hline IADL question (difficulty doing heavy housework) & Categorical \\
\hline IADL question (difficulty using public transportation) & Categorical \\
\hline Total MMSE score & Numeric \\
\hline $\begin{array}{l}\text { Sum of mobility score main features (em1,em2, } \\
\text { em3,em4,em5) }\end{array}$ & Numeric \\
\hline Number of drugs (drug intake) & Numeric \\
\hline Alkaline phosphatase [U/L] & Numeric \\
\hline Presence of polypharmacy & Binary \\
\hline Self-reported health status & Categorical \\
\hline $\begin{array}{l}\text { Self-reported health status compared to people the } \\
\text { same age }\end{array}$ & Categorical \\
\hline Capacity of dealing with problems & Categorical \\
\hline Capacity of dealing with tasks & Categorical \\
\hline GDS question (dropped activity of interests) & Binary \\
\hline GDS question (boredom) & Binary \\
\hline $\begin{array}{l}\text { Presence of joint inflammation (more than } 4 \text { weeks } \\
\text { in a row) }\end{array}$ & Categorical \\
\hline
\end{tabular}

variables that were present. Using a RF wrapper based feature selection method, potential predictors were identified. Further, previously known predictors for frailty, from the medical community, could be used to validate the built model and vice versa, the feature selection process confirmed their predictability. The present work has identified potential predictors for predicting frailty, which 
Table 3 10-fold cross-validation results for the binary classification models for each imputed data set, working with the two classes non - frail and frail

\begin{tabular}{|c|c|c|c|c|c|c|}
\hline Prediction method & Accuracy & AUC & Sensitivity & Specificity & Precision & $\mathrm{F}_{1}$-Score \\
\hline \multicolumn{7}{|l|}{ Imputation 1} \\
\hline Naive Bayes & $73.20 \pm 5.97 \%$ & $0.756 \pm 0.052$ & $0.656 \pm 0.102$ & $0.856 \pm 0.079$ & $0.885 \pm 0.054$ & $0.749 \pm 0.067$ \\
\hline CART & $72.77 \pm 5.20 \%$ & $0.710 \pm 0.061$ & $0.782 \pm 0.108$ & $0.639 \pm 0.168$ & $0.789 \pm 0.065$ & $0.778 \pm 0.049$ \\
\hline Bagging CART & $75.51 \pm 7.16 \%$ & $0.731 \pm 0.070$ & $0.830 \pm 0.086$ & $0.633 \pm 0.084$ & $0.786 \pm 0.048$ & $0.806 \pm 0.060$ \\
\hline C5.0 & $77.83 \pm 7.13 \%$ & $0.752 \pm 0.086$ & $0.860 \pm 0.056$ & $0.644 \pm 0.164$ & $0.804 \pm 0.075$ & $0.829 \pm 0.051$ \\
\hline Random forest & $77.64 \pm 5.62 \%$ & $0.755 \pm 0.053$ & $0.844 \pm 0.089$ & $0.667 \pm 0.087$ & $0.806 \pm 0.041$ & $0.823 \pm 0.050$ \\
\hline Support vector machines (RBF) & $77.64 \pm 6.55 \%$ & $0.762 \pm 0.065$ & $0.824 \pm 0.09$ & $0.700 \pm 0.099$ & $0.819 \pm 0.053$ & $0.819 \pm 0.057$ \\
\hline Linear discriminant analysis & $75.11 \pm 5.34 \%$ & $0.739 \pm 0.042$ & $0.789 \pm 0.096$ & $0.689 \pm 0.047$ & $0.805 \pm 0.023$ & $0.795 \pm 0.055$ \\
\hline \multicolumn{7}{|l|}{ Imputation 2} \\
\hline Naive Bayes & $72.78 \pm 6.47 \%$ & $0.750 \pm 0.059$ & $0.656 \pm 0.109$ & $0.844 \pm 0.094$ & $0.878 \pm 0.063$ & $0.745 \pm 0.072$ \\
\hline CART & $70.89 \pm 5.94 \%$ & $0.699 \pm 0.057$ & $0.741 \pm 0.098$ & $0.656 \pm 0.104$ & $0.781 \pm 0.047$ & $0.757 \pm 0.058$ \\
\hline Bagging CART & $75.11 \pm 6.59 \%$ & $0.729 \pm 0.072$ & $0.820 \pm 0.089$ & $0.639 \pm 0.134$ & $0.792 \pm 0.066$ & $0.802 \pm 0.054$ \\
\hline C5.0 & $77.39 \pm 7.35 \%$ & $0.745 \pm 0.093$ & $0.867 \pm 0.057$ & $0.622 \pm 0.192$ & $0.797 \pm 0.082$ & $\mathbf{0 . 8 2 8} \pm \mathbf{0 . 0 5 0}$ \\
\hline Random forest & $77.01 \pm 6.65 \%$ & $0.752 \pm 0.064$ & $0.827 \pm 0.101$ & $0.678 \pm 0.101$ & $0.809 \pm 0.052$ & $0.815 \pm 0.060$ \\
\hline Support vector machines (RBF) & $77.63 \pm 7.01 \%$ & $0.761 \pm 0.071$ & $0.827 \pm 0.085$ & $0.694 \pm 0.102$ & $0.816 \pm 0.057$ & $0.820 \pm 0.060$ \\
\hline Linear discriminant analysis & $76.14 \pm 5.15 \%$ & $0.752 \pm 0.046$ & $0.792 \pm 0.081$ & $0.711 \pm 0.057$ & $0.817 \pm 0.032$ & $0.803 \pm 0.050$ \\
\hline \multicolumn{7}{|l|}{ Imputation 3} \\
\hline Naive Bayes & $73.41 \pm 5.64 \%$ & $0.757 \pm 0.057$ & $0.664 \pm 0.083$ & $0.849 \pm 0.102$ & $0.885 \pm 0.069$ & $0.755 \pm 0.056$ \\
\hline CART & $73.21 \pm 5.75 \%$ & $0.728 \pm 0.07$ & $0.746 \pm 0.064$ & $0.709 \pm 0.14$ & $0.815 \pm 0.067$ & $0.776 \pm 0.045$ \\
\hline Bagging CART & $78.28 \pm 3.92 \%$ & $0.764 \pm 0.057$ & $0.841 \pm 0.058$ & $0.688 \pm 0.148$ & $0.823 \pm 0.062$ & $0.828 \pm 0.026$ \\
\hline C5.0 & $74.06 \pm 7.12 \%$ & $0.709 \pm 0.089$ & $0.837 \pm 0.057$ & $0.581 \pm 0.181$ & $0.774 \pm 0.073$ & $0.802 \pm 0.048$ \\
\hline Random forest & $77.62 \pm 6.65 \%$ & $0.762 \pm 0.076$ & $0.820 \pm 0.068$ & $0.704 \pm 0.134$ & $0.824 \pm 0.068$ & $0.820 \pm 0.052$ \\
\hline Support vector machines (RBF) & $79.32 \pm 5.00 \%$ & $0.779 \pm 0.056$ & $0.838 \pm 0.049$ & $0.720 \pm 0.09$ & $0.833 \pm 0.048$ & $\mathbf{0 . 8 3 4} \pm \mathbf{0 . 0 4 0}$ \\
\hline Linear discriminant analysis & $78.47 \pm 4.77 \%$ & $0.773 \pm 0.051$ & $0.821 \pm 0.059$ & $0.726 \pm 0.085$ & $0.833 \pm 0.045$ & $0.825 \pm 0.040$ \\
\hline \multicolumn{7}{|l|}{ Imputation 4} \\
\hline Naive Bayes & $72.78 \pm 5.89 \%$ & $0.750 \pm 0.061$ & $0.657 \pm 0.083$ & $0.843 \pm 0.111$ & $0.881 \pm 0.075$ & $0.749 \pm 0.057$ \\
\hline CART & $71.26 \pm 5.83 \%$ & $0.697 \pm 0.053$ & $0.762 \pm 0.095$ & $0.631 \pm 0.083$ & $0.774 \pm 0.043$ & $0.765 \pm 0.058$ \\
\hline Bagging CART & $76.38 \pm 5.77 \%$ & $0.747 \pm 0.069$ & $0.817 \pm 0.076$ & $0.676 \pm 0.147$ & $0.812 \pm 0.065$ & $0.811 \pm 0.046$ \\
\hline C5.0 & $74.25 \pm 7.13 \%$ & $0.712 \pm 0.085$ & $0.837 \pm 0.057$ & $0.587 \pm 0.157$ & $0.774 \pm 0.07$ & $0.803 \pm 0.052$ \\
\hline Random forest & $76.99 \pm 5.90 \%$ & $0.755 \pm 0.069$ & $0.817 \pm 0.069$ & $0.693 \pm 0.136$ & $0.819 \pm 0.067$ & $0.815 \pm 0.046$ \\
\hline Support vector machines (RBF) & $78.47 \pm 5.14 \%$ & $0.771 \pm 0.057$ & $0.827 \pm 0.053$ & $0.714 \pm 0.092$ & $0.829 \pm 0.049$ & $0.827 \pm 0.041$ \\
\hline Linear discriminant analysis & $78.06 \pm 5.39 \%$ & $0.772 \pm 0.057$ & $0.807 \pm 0.061$ & $0.737 \pm 0.091$ & $0.837 \pm 0.049$ & $0.820 \pm 0.045$ \\
\hline \multicolumn{7}{|l|}{ Imputation 5} \\
\hline Naive Bayes & $73.41 \pm 5.45 \%$ & $0.756 \pm 0.053$ & $0.664 \pm 0.088$ & $0.849 \pm 0.098$ & $0.885 \pm 0.066$ & $0.754 \pm 0.057$ \\
\hline CART & $71.67 \pm 7.79 \%$ & $0.702 \pm 0.087$ & $0.762 \pm 0.100$ & $0.642 \pm 0.166$ & $0.786 \pm 0.089$ & $0.769 \pm 0.066$ \\
\hline Bagging CART & $76.79 \pm 4.69 \%$ & $0.749 \pm 0.053$ & $0.827 \pm 0.071$ & $0.671 \pm 0.115$ & $0.809 \pm 0.049$ & $0.815 \pm 0.039$ \\
\hline C5.0 & $75.31 \pm 4.08 \%$ & $0.726 \pm 0.055$ & $0.837 \pm 0.065$ & $0.615 \pm 0.138$ & $0.787 \pm 0.055$ & $0.808 \pm 0.030$ \\
\hline Random forest & $78.03 \pm 5.10 \%$ & $0.764 \pm 0.060$ & $0.830 \pm 0.073$ & $0.698 \pm 0.129$ & $0.824 \pm 0.061$ & $0.824 \pm 0.041$ \\
\hline Support vector machines (RBF) & $78.47 \pm 5.39 \%$ & $0.771 \pm 0.059$ & $0.827 \pm 0.055$ & $0.714 \pm 0.092$ & $0.828 \pm 0.049$ & $0.827 \pm \mathbf{0 . 0 4 3}$ \\
\hline Linear discriminant analysis & $77.62 \pm 5.35 \%$ & $0.769 \pm 0.058$ & $0.800 \pm 0.063$ & $0.737 \pm 0.102$ & $0.836 \pm 0.054$ & $0.816 \pm 0.045$ \\
\hline
\end{tabular}

The highest obtained value for each performance category for each imputed data set is marked in bold 
were conformed by the doctors. Most of the found predictors are variables describing the mobility, the mental state and the capability of performing daily tasks.

Some interesting findings, according to the physicians is for example the presence of blood alkaline phosphatase level in $U / L$ in the selected feature set (Table 2). Less surprising is that age is also among these features. Moreover, the final feature set also included variables regarding: depression (presence of depression, total GDS, $2^{\text {nd }}$ GDS question, $4^{\text {th }}$ GDS question), polypharmacy (presence of polypharmacy, number of drugs), mobility (mobility score), Mini-Mental-State-Examination (total MMSE score, presence of cognitive impairment, MMSE followup question [remembering objects ability]), Instrumental Activities of Daily Living (number of IADL abilities and the first 6 IADL questions), Activities of Daily Living (number of ADL abilities and ADL question [difficulty washing]), self-reported health-status (self-reported health status, self-reported health status compared to people the same age, capacity of dealing with problems, capacity of dealing with tasks) and rheumatic disease (presence of joint inflammation [more than 4 weeks in a row]), which also according to the doctors seem to be relevant.

The found feature set seems to be consistent with known frailty risk factors or preventive factors found by the medical community. Interesting seems to be the finding that the feature $p 40 \mathrm{falc}$, representing the blood alkaline phosphatase level in U/L, is highly predictive. This certainly requires some follow up investigations, as this could possibly be a new biomarker for frailty detection. The doctors said that this variable is probably a good predictor, because it gives information about inflammation processes in the body. They are already investigating it, in the scope of the FRAILOMIC initiative [46], which is a research project aiming to identify the factors that turn frailty into disability. The doctors conformed that the found predictors are related to frailty. They commented also on the missingness of the gender feature. According to them, it's one of the important markers for determining frailty and they were surprised that it did not appear in the final predictor set. It is possible that the feature selection algorithm found this variable to be redundant and that the contained information is already provided by other features. The variable height is, for example, highly correlated to the gender variable (correlation coefficient $=0.725$ ). This manifests that further analysis with a bigger population is required in order to understand the role of this variable in particular but also for all the found potential predictors.

The set presented, results to be the best subset of features for the task of predicting frailty, even that some of these variables showed to be correlated in the data understanding phase. Machine learning algorithms are very flexible with regard to problems of multicollinearity, especially tree based ones and the SVM [47, 48]. In fact, that are the methods which were used for the predictive modeling. Consequently, the possible collinearity impact on the prediction models is avoided.

\section{Evaluation of the built models}

For this research two different evaluations are required. First, the analyses of the performances of the models and later, the analysis of how the models actually fit the goals. The overall best performances in nearly all measures have SVMs with a RBF as kernel. Followed by RF, LDA, bagging CART, C5.0, NB and CART. Striking is the high obtained specificity and precision of the NB classifier, while it performs inferior in the other measures compared to the other models. In this case specificity represents the ratio of predicted real non-frail patients to all non-frail patients. Thus, this classifier shows an extraordinary performance in the task of detecting non-frail patients. The highest values for accuracy and AUC are always achieved by RF and SVMs, which do not differ significantly in their results. The highest scores in each category for each imputation are marked in bold in Table 3. The variation of the results between the different imputed data sets is also very small, which indicates that also the variation of the imputed values is small. For example, the accuracy of SVM averaged over all imputed data sets is $78.31 \pm 0.70 \%$. The standard deviation is not even one percent. The RF algorithm performed slightly inferior with an averaged accuracy of $77.46 \pm 0.45 \%$. Here the standard deviation is below a half percent, which shows that the performance is quite stable.

The built models achieved an accuracy of more than $78 \%$ for binary classification of the frailty variable, without using features, which are directly related to the target or used to build it (see Fried's frailty criteria and stages [5]). The results show, that it is feasible to build predictive models for the frailty syndrome using medical data.

\section{Interpretability of the built models}

The tree models derived by CART and C5.0 are easy to interpret as they can provide "human-friendly" explanations, which is an extremely important aspect (see e.g. [49]). Tree structures are ideal for capturing interactions between the features and present themselves a natural visualization with edges and nodes. Hence, good explanations could be derived, although linear relationships are presented by splits. When bagging is used (bagging CART, RF), the resulting model is not a single tree but an ensemble of trees, which significantly decreases the interpretability. NB is a simple and interpretable model. The contribution of each feature towards the final chosen class is clear. LDA also provides a result, which is very easy to interpret, as the output is a linear combination of the 
features. In case of the SVM the interpretability depends on the chosen kernel. If a non linear kernel is used, as in this work, the relationships can not be easily captured.

\section{Limitations}

Predictive models, using the predictors obtained in the feature selection process, were built in order to predict frailty in patients. It was decided to derive a binary classifier, which is able to separate the two classes non-frail and frail. The classes pre-frail and frail from the original multiclass problem were fused into the class frail in order to work on a binary classification problem. Even though that could cause a degradation of the performance of the built models.

Further, it has to be stated that the derived model is technically speaking a predictive model, but presents semantically a diagnostic model. As already mentioned, temporal analysis was not possible as mainly data from one point in time was available. Thus, the built model relies only on data which has been collected at the same time as the diagnosis has been made.

By using multiple 10-fold cross-validations, a first estimate of the generalization error is obtained. Though, according to Bellazzi et al. (2011) [50] the prediction performance should also be tested on an independent data test set from another study.

Regarding the performed imputations of missing values, one could argue that using correlation between variables as a criteria for the imputation process could boost existing correlations, however multiple imputations have been used to reflect the degree of uncertainty when making use of such an approach and therefore not an exact result but an performance estimate has been presented.

Null imputation is a task that on its own requires a lot of work due to the vast amount of decisions that have to be made. In fact for each attribute a deep analysis is required. In this work 157 attributes are given for which data imputation is required. Due to the fact that the main goal of the present work is showing that prediction of frailty is feasible rather than analyzing the most efficient algorithm for a prediction, quite enough effort has been dedicated to null imputation. However, a deeper analysis would be needed in order to answer questions related to the statistical analysis of the multiple imputations and also to the obtained statistical results, which are pooled into a final point estimate plus standard error, applying Rubin's pooling rules [51].

It is also important noting that several issues make medical data mining a hard task today. On the one hand, problems related to legal issues and all the issues concerning privacy and confidentiality and on the other hand, the problem of interoperability of systems make it difficult to have a complete view of the patient or to integrate data from different services at the hospital. Besides, one cannot forget the effort of obtaining a complete cohort of patients from which we can extract results. Consequently, in this work we would only analyze a cohort of 474 patients for which 284 variables were available. These data limitations allowed for only rough performance estimates for the models. It would be desirable to have a bigger sample, so that results would become more significant and validations would be possible in different cohorts.

\section{Conclusions}

In this paper the feasibility of applying data mining techniques in order to extract models for frailty prediction using medical data from patients some of which are frail, has been analyzed.

From the work developed, it has been shown that in fact it is possible to extract meaningful patterns. Further, the importance of data preparation and data understanding for the successful extraction of predictive patterns has been demonstrated. Despite the importance of intelligent algorithms to extract the patterns, in this work we have additionally shown the paramount importance of pre-processing. Without a modest amount of effort in this phase, a reliable prediction model can not be built. Therefore, investing a lot of work here proved to be highly beneficial in terms of accuracy and reliability of the obtained predictions.

\section{Future work}

This work contributed towards obtaining predictive models that can anticipate the onset of age related deterioration. In particular, the problem of frailty has been analyzed in this paper. However, for these models to be used in daily routine, some work still needs to be done, nevertheless, this work opens new lines of research.

A next step is to analyze the best algorithm depending on the size of the data set. In this work the main focus was to show that data analysis is possible rather than showing which methods are the most efficient. Consequently, in future work the feature selection process should be repeated once data of more patients is available. Moreover, we have focused on obtaining models for a binary variable FRAILTY, but in the future more models should be created in order to analyze differences between the the stages non-frail and pre-frail, and pre-frail and frail respectively.

All in all, one remaining task is removing step by step the expert from the deep processes of the data preparation pipeline by further developing the autonomy of the system. Yet, according to Holzinger (2017) [52] it seems to be unrealistic that such fully automatic approaches can be realized in the near future, most of all it is very important in the medical domain to foster transparency and trust. Standard black-box approaches lack transparency, hence do not foster trust and acceptance. Rising legal and privacy aspects, e.g. with the new European General Data 
Protection Regulations will make it more important in the future to explain why a decision has been made [53]. So in contrast to pursue the objective of increasing the autonomy of the process, it should be considered to include doctors as agents in the development of the predictive algorithm, in order make their domain-knowledge during the learning process available, which potentially could increase the performance of the final model [52]. Maybe in a more distant future fully automatic approaches will be feasible.

Besides, future research will include analyzing more features. In particular investigating the impact of medication or the impact of nutritional information is very promising. Moreover, future work could also focus on evolution analysis of patients regarding the frailty syndrome. The most important future work is to validate the results in other cohorts and it is necessary to check how the model

Albeit the results seem to be very promising, for them to have more impact, it would be required to further validate the results in other cohorts, along with testing on how the derived model from retrospective data performs in prospective trials.

\section{Additional file}

Additional file 1: Data Dictionary: Description of the Variables. This file "Additional File 1.pdf" contains tables where for each available variable the name, expected values, a description and the data type is stated. The variables where divided into semantic groups, where for each group a table has been created. These groups have already been mentioned in the subsection Definition of the Variables. (PDF $93 \mathrm{~kb}$ )

\section{Abbreviations}

ADL: Activities of daily living; AUC: Area under the curve; CART: Classification and regression trees; CDSS: Clinical decision support system; DALY: Disability adjusted life year; DHEAS: Dehydroepiandrosterone sulfate; GDS: Geriatric depression scale; IADL: Instrumental activities of daily living; iML: Interactive machine learning; LDA: Linear discriminant analysis; MAR: Missing at random MCAR: Missing completely at random; MMSE: Mini-mental-state-examination; MNAR: Missing not at random; MS: Mobility scale; NA: Not available; NB: Naïve Bayes; NN: Neural network; PASE: Physical activity scale for the elderly; RBF: Radial basis function; RF: Random forest; SVM: Support vector machine; TSHA: Toledo study for healthy aging

\section{Acknowledgements}

The authors are grateful to Dr. Ángel García-Pedrero and Juan Manuel Tuñas Martín and also to the rest of the team of the MEDAL Laboratory of the Center of Biomedical Technology (CTB) of the Universidad Politécnica de Madrid (UPM) for their help and their insightful comments.

\section{Funding}

The work has been partially financed under the project FACET (EIT-HEALTH).

\section{Availability of data and materials}

The data are available from from the authors upon reasonable request for research and with permission of Complejo Hospitalario de Toledo and Hospital Universitario de Getafe.

\section{Authors' contributions}

All authors contributed in writing this article. EM proposed developing a binary classifier for the diagnosing of frail patients using the Toledo Study for
Healthy Aging (TSHA) data. Further, EM helped with the development of the methodology. FJGG provided the TSHA study data and gave feedback throughout the process. LRM helped with interpreting the medical data and providing feedback for the obtained results from the medical perspective. APH designed, implemented and validated the methodology, supervised by $\mathrm{AH}$ and EM. All authors read and approved the final manuscript.

\section{Ethics approval and consent to participate}

The TSHA has been approved by the Ethic Committee of both Complejo Hospitalario de Toledo and Hospital Universitario de Getafe. All individuals participating in this study provided full written informed consent to have their samples and data used for research purposes, including genetic ones (although this is not applicable in this case).

\section{Consent for publication}

Not applicable.

\section{Competing interests}

The authors declare that they have no competing interests. AH is an Editorial Board Member for BMC Medical Informatics and Decision Making.

\section{Publisher's Note}

Springer Nature remains neutral with regard to jurisdictional claims in published maps and institutional affiliations.

\section{Author details}

${ }^{1}$ Holzinger Group, HCI-KDD, Institute for Medical Informatics/Statistics, Medical University Graz, 8036 Graz, Austria. ${ }^{2}$ Institute of Interactive Systems and Data Science, Graz University of Technology, $8010 \mathrm{Graz}$, Austria. ${ }^{3}$ Center for Biomedical Technology, Universidad Politecnica de Madrid, 28000 Madrid, Spain. ${ }^{4}$ Division of Geriatric Medicine, Virgen del Valle Geriatric Hospital, 45000 Toledo, Spain. ${ }^{5}$ Division of Geriatric Medicine, University Hospital of Getafe, 28905 Getafe, Spain.

Received: 19 September 2017 Accepted: 18 January 2019

Published online: 18 February 2019

\section{References}

1. Economic Policy Committee and others. The 2009 Ageing Report: economic and budgetary projections for the EU-27 Member States (2008-2060). European Economy. 2009;2. https://doi.org/10.2765/80301.

2. Murray CJ, Vos T, Lozano R, Naghavi M, Flaxman AD, Michaud C, Ezzati M, Shibuya K, Salomon JA, Abdalla S, et al. Disability-adjusted life years (dalys) for 291 diseases and injuries in 21 regions, 1990-2010: a systematic analysis for the global burden of disease study 2010. Lancet. 2013;380(9859):2197-223.

3. Kleinberger T, Becker M, Ras E, Holzinger A, Müller P. Ambient intelligence in assisted living: Enable elderly people to handle future interfaces. 2007103-112. https://doi.org/10.1007/978-3-540-73281-5_11.

4. Bellazzi R, Zupan B. Predictive data mining in clinical medicine: Current issues and guidelines. Int J Med Inform. 2008;77(2):81-97. https://doi.org/ 10.1016/j.ijmedinf.2006.11.006.

5. Fried LP, Tangen CM, Walston J, Newman AB, Hirsch C, Gottdiener J, Seeman T, Tracy R, Kop WJ, Burke G, McBurnie MA. Frailty in older adults: Evidence for a phenotype. J Gerontol A Biol Sci Med Sci. 2001;56(3):146-57. https://doi.org/10.1093/gerona/56.3.m146.

6. Rodriguez-Mañas L, Fried LP. Frailty in the clinical scenario. Lancet. 2015;385(9968):7-9. https://doi.org/10.1016/s0140-6736(14)61595-6.

7. Safran C, Bloomrosen M, Hammond WE, Labkoff S, Markel-Fox S, Tang PC, Detmer DE. Toward a national framework for the secondary use of health data: An american medical informatics association white paper. J Am Med Inform Assoc. 2007;14(1):1-9. https://doi.org/10.1197/jamia. m2273.

8. Jaspers MWM, Smeulers M, Vermeulen H, Peute LW. Effects of clinical decision-support systems on practitioner performance and patient outcomes: a synthesis of high-quality systematic review findings. J Am Med Inform Assoc. 2011;18(3):327-34. https://doi.org/10.1136/amiajnl2011-000094.

9. Bright TJ, Wong A, Dhurjati R, Bristow E, Bastian L, Coeytaux RR, Samsa G, Hasselblad V, Williams JW, Musty MD, et al. Effect of clinical decision-support systemsa systematic review. Ann Intern Med. 2012;157(1):29-43. 
10. Bose NK, Liang P. Neural network fundamentals with graphs, algorithms, and applications. New York: McGraw-Hill Inc.; 1996.

11. Schmidhuber J. Deep learning in neural networks: An overview. Neural Netw. 2015;61:85-117.

12. Rish I, et al. An empirical study of the naive bayes classifier. In: IJCAI 2001 Workshop on Empirical Methods in Artificial Intelligence, vol 3. New York: IBM; 2001. p. 41-6.

13. Izenman AJ. Linear Discriminant Analysis. In: Modern Multivariate Statistical Techniques: Regression, Classification, and Manifold Learning. New York: Springer; 2008. p. 237-80. https://doi.org/10.1007/978-0-38778189-1_8.

14. Hearst MA, Dumais ST, Osuna E, Platt J, Scholkopf B. Support vector machines. IEEE Intell Syst Appl. 1998;13(4):18-28. https://doi.org/10.1109/ 5254.708428 .

15. Breiman L. Classification and Regression Trees; 2017. https://doi.org/10. 1201/9781315139470. https://doi.org/10.1201\%2F9781315139470.

16. Holzinger A. Interactive machine learning for health informatics: when do we need the human-in-the-loop? Brain Inform. 2016;3(2):119-31.

17. Ensrud KE, Ewing SK, Taylor BC, et al. Comparison of 2 frailty indexes for prediction of falls, disability, fractures, and death in older women. Arch Intern Med. 2008;168(4):382-9. https://doi.org/10.1001/archinternmed. 2007.113.

18. Drubbel I, de Wit NJ, Bleijenberg N, Eijkemans RJC, Schuurmans MJ, Numans ME. Prediction of adverse health outcomes in older people using a frailty index based on routine primary care data. J Gerontol A. 2013;68(3):301-8. https://doi.org/10.1093/gerona/gls161.

19. Fried LP, Ferrucci L, Darer J, Williamson JD, Anderson G. Untangling the concepts of disability, frailty, and comorbidity: Implications for improved targeting and care. J Gerontol A. 2004;59(3):255-63. https://doi.org/10. 1093/gerona/59.3.M255.

20. Makary MA, Segev DL, Pronovost PJ, Syin D, Bandeen-Roche K, Patel P, Takenaga R, Devgan L, Holzmueller CG, Tian J, et al. Frailty as a predictor of surgical outcomes in older patients. J Am Coll Surg. 2010;210(6):901-8.

21. Kim SW, Han HS, Jung HW, Kim Kl, Hwang DW, Kang SB, Kim CH. Multidimensional frailty score for the prediction of postoperative mortality risk. JAMA Surg. 2014;149(7):633-40. https://doi.org/10.1001/ jamasurg.2014.241. /data/journals/surg/930571/soi140018.pdf.

22. Fiatarone MA, O'neill EF, Ryan ND, Clements KM, Solares GR, Nelson ME, Roberts SB, Kehayias JJ, Lipsitz LA, Evans WJ. Exercise training and nutritional supplementation for physical frailty in very elderly people. $\mathrm{N}$ Engl J Med. 1994;330(25):1769-75. https://doi.org/10.1056/ NEJM199406233302501.

23. Wolf SL, Barnhart HX, Kutner NG, McNeely E, Coogler C, Xu T. Reducing frailty and falls in older persons: An investigation of tai chi and computerized balance training. J Am Geriatr Soc. 44(5):489-497. https:// doi.org/10.1111/j.1532-5415.1996.tb01432.x. https://onlinelibrary.wiley. com/doi/pdf/10.1111/j.1532-5415.1996.tb01432.x.

24. Collard RM. Frailty \& late-life depression: a delicate balance. Radboud University Nijmegen; 2015. https://repository.ubn.ru.nl/handle/2066/ 144024.

25. Bouillon K, Kivimäki M, Hamer M, Shipley MJ, Akbaraly TN, Tabak A, Singh-Manoux A, Batty GD. Diabetes risk factors, diabetes risk algorithms, and the prediction of future frailty: The whitehall ii prospective cohort study. J Am Med Dir Assoc. 2013;14(11):851-18516. https://doi.org/10. 1016/j.jamda.2013.08.016.

26. Bandeen-Roche K, Xue Q-L, Ferrucci L, Walston J, Guralnik JM, Chaves P, Zeger SL, Fried LP. Phenotype of frailty: Characterization in the women's health and aging studies. J Gerontol A. 2006;61(3):262-6. https://doi.org/ 10.1093/gerona/61.3.262.

27. Walston J, McBurnie M, Newman A, et al. Frailty and activation of the inflammation and coagulation systems with and without clinical comorbidities: Results from the cardiovascular health study. Arch Intern Med. 2002;162(20):2333-41. https://doi.org/10.1001/archinte.162.20.2333.

28. Baylis D, Bartlett DB, Syddall HE, Ntani G, Gale CR, Cooper C, Lord JM, Sayer AA. Immune-endocrine biomarkers as predictors of frailty and mortality: a 10-year longitudinal study in community-dwelling older people. AGE. 2013;35(3):963-71. https://doi.org/10.1007/s11357-0129396-8.

29. Garcia-Garcia FJ, Avila GG, Alfaro-Acha A, Andres MSA, de la Torre Lanza MDLA, Aparicio MVE, Aparicio SH, Zugasti JLL, Reus MG-S, Rodriguez-Artalejo F, Rodriguez-Manas L. The prevalence of frailty syndrome in an older population from spain. the toledo study for healthy aging. J Nutr, Health Aging. 2011;15(10):852-6. https://doi.org/10.1007/ s12603-011-0075-8.

30. Yesavage JA, Sheikh Jl. 9/geriatric depression scale (GDS). Clin Gerontol. 1986;5(1-2):165-73.

31. Yesavage JA, Brink TL, Rose $T L$, Lum O, Huang V, Adey M, Leirer VO. Development and validation of a geriatric depression screening scale: a preliminary report. J Psychiatr Res. 1983;17(1):37-49.

32. KATZ S. Assessing self-maintenance: Activities of daily living, mobility, and instrumental activities of daily living. J Am Geriatr Soc. 1983;31(12):721-7. https://doi.org/10.1111/j.1532-5415.1983.tb03391.x.

33. Lawton MP, Brody EM. Assessment of older people: self-maintaining and instrumental activities of daily living. Nurs Res. 1970;19(3):278. https://doi. org/10.1097/00006199-197005000-00029.

34. Folstein MF, Folstein SE, McHugh PR. "mini-mental state": a practical method for grading the cognitive state of patients for the clinician. J Psychiatr Res. 1975;12(3):189-98.

35. Cockrell JR, Folstein MF. Mini-mental state examination. Principles and practice of geriatric psychiatry. 2002;140-141. https://doi.org/10.1002/ 0470846410.ch27(ii).

36. Washburn RA, Smith KW, Jette AM, Janney CA. The physical activity scale for the elderly (PASE): Development and evaluation. J Clin Epidemiol. 1993;46(2):153-62. https://doi.org/10.1016/0895-4356(93)90053-4.

37. Wartner S, Girardi D, Wiesinger-Widi M, Trenkler J, Kleiser R, Holzinger A. Ontology-guided principal component analysis: Reaching the limits of the doctor-in-the-loop. In: International Conference on Information Technology in Bio-and Medical Informatics. Springer; 2016. p. 22-33. https://doi.org/10.1007/978-3-319-43949-5_2. https://doi.org/10.1007 \%2F978-3-319-43949-5_2.

38. Agrawal R, Mannila H, Srikant R, Toivonen $H$, Verkamo Al, et al. Fast discovery of association rules. Adv Knowl Disc Data Min. 1996;12(1):307-28.

39. Eknoyan Garabed. Adolphe Quetelet (1796-1874)-the average man and indices of obesity. Nephrol Dial Transplant. 2007;23(1):47-51.

40. Shah AD, Bartlett JW, Carpenter J, Nicholas O, Hemingway $H$. Comparison of random forest and parametric imputation models for imputing missing data using MICE: A CALIBER study. Am J Epidemiol. 2014;179(6):764-74. https://doi.org/10.1093/aje/kwt312.

41. van Buuren S, Groothuis-Oudshoorn K. mice: Multivariate imputation by chained equations in r. J Stat Softw. 2011;45(3):. https://doi.org/10.18637/ jss.v045.i03.

42. Kursa MB, Rudnicki WR. Feature selection with the boruta package. J Stat Softw. 2010;36(11):1-13.

43. Le J. The 10 algorithms machine learning engineers need to know. KDnuggets Analytics Big Data Data Min Data Sci. 2016. https://www. kdnuggets.com/2016/08/10-algorithms-machine-learning-engineers. html. Accessed 15 Aug 2017.

44. Bradley AP. The use of the area under the roc curve in the evaluation of machine learning algorithms. Pattern Recogn. 1997;30(7):1145-59.

45. Sokolova M, Lapalme G. A systematic analysis of performance measures for classification tasks. Inf Process Manag. 2009;45(4):427-37.

46. Lippi G, Jansen-Duerr P, Viña J, Durrance-Bagale A, Abugessaisa I, Gomez-Cabrero D, Tegnér J, Grillari J, Erusalimsky J, Sinclair A, Rodriguez-Manãs L, on behalf of the FRAILOMIC consorti. Laboratory biomarkers and frailty: presentation of the FRAILOMIC initiative. Clin Chem Lab Med (CCLM). 2015;53(10):. https://doi.org/10.1515/cclm-2015-0147.

47. Caraviello D, Weigel K, Craven M, Gianola D, Cook N, Nordlund K, Fricke P, Wiltbank M. Analysis of reproductive performance of lactating cows on large dairy farms using machine learning algorithms. J Dairy Sci. 2006;89(12):4703-22.

48. Kotsiantis SB, Zaharakis I, Pintelas P. Supervised machine learning: A review of classification techniques. In: Proceedings of the 2007 Conference on Emerging Artificial Intelligence Applications in Computer Engineering: Real Word Al Systems with Applications in eHealth, $\mathrm{HCl}$, Information Retrieval and Pervasive Technologies. Amsterdam: IOS Press; 2007. p. 3-24. http://dl.acm.org/citation.cfm?id=1566770.1566773.

49. Hudec M, Bednárová E, Holzinger A. Augmenting statistical data dissemination by short quantified sentences of natural language. J Off Stat (JOS). 2018;34(4):981. https://doi.org/10.2478/jos-2018-0048.

50. Bellazzi R, Ferrazzi F, Sacchi L. Predictive data mining in clinical medicine: a focus on selected methods and applications. Wiley Interdiscip Rev Data Min Knowl Disc. 2011;1(5):416-30. https://doi.org/10.1002/widm.23.

51. Buuren S. Flexible Imputation of Missing Data; 2012. https://doi.org/10. 1201/b1 1826. https://doi.org/10.1201\%2Fb11826. 
52. Holzinger A. Introduction to machine learning \& knowledge extraction (make). In: Machine Learning and Knowledge Extraction, vol 1; 2017. p. 1-20. https://doi.org/10.3390/make1010001.

53. Holzinger A, Plass M, Kickmeier-Rust M, Holzinger K, Crişan GC, Pintea C-M, Palade V. Interactive machine learning: experimental evidence for the human in the algorithmic loop. Appl Intell. 2018. https://doi.org/10. 1007/s10489-018-1361-5.

54. Kursa MB, Jankowski A, Rudnicki WR. Boruta-a system for feature selection. Fundam Informaticae. 2010;101(4):271-85.

Ready to submit your research? Choose BMC and benefit from:

- fast, convenient online submission

- thorough peer review by experienced researchers in your field

- rapid publication on acceptance

- support for research data, including large and complex data types

- gold Open Access which fosters wider collaboration and increased citations

- maximum visibility for your research: over $100 \mathrm{M}$ website views per year

At BMC, research is always in progress.

Learn more biomedcentral.com/submissions 